Steve HAGIMONT, Commercialiser la nature et les façons d'être. Une histoire sociale et environnementale de l'économie et de l'aménagement touristiques (Pyrénées françaises et espagnoles, $X I X^{e}-X X^{e}$ siècles)

Thèse d'histoire réalisée à l'Université de Toulouse sous la direction de Jean-Michel MINOVEZ et Vincent VLÈs, soutenue le 25 novembre 2017, 3 volumes, $1210 \mathrm{p}$.

The Commodification of Nature and Human Existences. A Social and Environmental History of Tourist Economy and Planning (French and Spanish Pyrenees, XIXth-XXth centuries)

\title{
Steve Hagimont
}

\section{OpenEdition} Journals

Édition électronique

URL : http://journals.openedition.org/tourisme/1845

DOI : 10.4000/tourisme.1845

ISSN : 2492-7503

Éditeur

Éditions touristiques européennes

Référence électronique

Steve Hagimont, «Steve HAGIMON, Commercialiser la nature et les façons d'être. Une histoire sociale et environnementale de l'économie et de l'aménagement touristiques (Pyrénées françaises et espagnoles, XIXe XXe siècles) », Mondes du Tourisme [En ligne], 14 | 2018, mis en ligne le 30 juin 2018, consulté le 25 septembre 2020. URL : http://journals.openedition.org/tourisme/1845 ; DOI : https://doi.org/10.4000/ tourisme.1845

Ce document a été généré automatiquement le 25 septembre 2020 


\section{Steve HAGIMONT, Commercialiser la} nature et les façons d'être. Une histoire sociale et environnementale de l'économie et de l'aménagement touristiques (Pyrénées françaises et espagnoles, $\mathrm{XIX}^{e}-\mathrm{XX} \mathrm{X}^{e}$ siècles)

Thèse d'histoire réalisée à l'Université de Toulouse sous la direction de Jean-Michel MINOVEZ et Vincent VLÈs, soutenue le 25 novembre 2017, 3 volumes, $1210 \mathrm{p}$.

The Commodification of Nature and Human Existences. A Social and Environmental History of Tourist Economy and Planning (French and Spanish Pyrenees, XIXth-XXth centuries)

\section{Steve Hagimont}

1 Ce travail examine l'appropriation locale d'un changement global d'imaginaire, qui transforme les montagnes, leur environnement et leurs collectifs humains en objets de désirs et de consommations touristiques. À partir de la fin du XVIII ${ }^{\mathrm{e}}$ siècle se construit en effet, d'abord localement, un marché touristique international. Un marché qui naît de la prise de conscience que ces désirs de nature sont une source de profit potentielle, dans des espaces montagnards trop souvent considérés comme coupés du reste du monde. Suivant les perspectives théoriques ouvertes par Cornelius Castoriadis et Maurice Godelier, ces travaux veulent inscrire dans l'étude historique de l'économie, communément matérielle, la part indissociable d'imaginaire et de symbolique qui motive, donne sens et reconduit des dispositifs tout à fait matériels. Cette étude s'inscrit dans le temps long (entre la fin du XVIII ${ }^{\mathrm{e}}$ et la fin du XX $\mathrm{X}^{\mathrm{e}}$ siècle) et s'appuie sur un espace pionnier du tourisme européen : les Pyrénées. La chronologie débute de la fin 
du XVIII siècle, lorsqu'émergent ces rêves de nature et les premiers aménagements d'envergure pour les faire fructifier (qu'émergent en fait de premières "stations »), et s'étend jusqu'à la remise en cause des logiques d'aménagement «industriel» de la montagne, qui se manifeste autour de 1970 et se concrétise dans la loi dite «montagne » de 1985.

2 Les archives, particulièrement riches, de l'aménagement et de l'économie touristiques, permettent de retracer les actions entreprises pour construire une offre touristique efficace, les acteurs, leurs logiques et les conflits engendrés. Au XVIII ${ }^{\mathrm{e}}$ siècle donc, avant même que l'industrialisation n'entre dans les consciences communes, des désirs de nature se diffusent, s'incarnent dans les montagnes et transforment certains de leurs éléments (leurs eaux, leur air, leurs neiges, leur lumière, leurs paysages) en sources d'émotions, de santé et de repos dignes de déplacements et de consommations. Les Pyrénées sont prises dans ce mouvement imaginaire; de nouvelles ressources, touristiques, y naissent ainsi. Des acteurs d'origine diverse s'ingénient dès lors à les transformer en produits de consommation, par les thermes, l'urbanisme, les hôtels, les villas, les promenades, les routes d'abord, puis par les sentiers, les refuges, les chemins de fer et, plus tard encore, par les stations de sports d'hiver à partir des années 1910. Des produits proposés sur un marché de plus en plus concurrentiel et diversifié. Ainsi, ces montagnes, représentées comme en marge des transformations économiques et sociales connues depuis la fin du XVIII e siècle en Europe, sont en fait l'un des cœurs de «la » modernité. Accompagnant, voire devançant, l'industrialisation en fin de XVIII ${ }^{\mathrm{e}}$ siècle, le tourisme permet l'intégration précoce de certaines zones à la marche la plus contemporaine de l'économie. Au cours des $\mathrm{XIX}^{\mathrm{e}}$ et $\mathrm{XX}^{\mathrm{e}}$ siècles, ces espaces montagnards touristiques deviennent des compensations indispensables aux " excès » environnementaux, politiques, sociaux et identitaires de la société urbaine et industrielle. À chaque moment de cette histoire du tourisme, certaines régions des Pyrénées se situent au meilleur niveau d'équipement et de réputation. Luchon ou Cauterets au XIX ${ }^{\mathrm{e}}$ siècle, le Val d'Aran (Espagne) ou l'Andorre au $\mathrm{XX}^{\mathrm{e}}$ siècle apparaissent donc comme des espaces essentiels de la construction et du renouvellement économique, social, culturel et environnemental des sociétés occidentales contemporaines.

3 Le tourisme fait donc advenir en tant que produits de consommation ludiques et sanitaires des éléments humains et non humains puisés dans les montagnes. Dans ce processus, les populations locales ne sont pas des spectateurs passifs: elles sont motrices de la mise en tourisme de leurs propres vies et de leurs environnements quotidiens et, ce, dès le départ. Bien sûr, l'opposition entre le local et l'extérieur est avant tout un ressort discursif; dans les faits, les échelles d'appartenance se brouillent. L'ancrage local n'empêche en tout cas aucunement de tisser des réseaux d'échelle régionale et nationale; l'ancrage local n'empêche pas non plus des circulations européennes de modèles d'aménagement, comme le montre le cas des thermes, des casinos, des règlements d'urbanisme et de police au XIX siècle ou des stations de sports d'hiver au XXe. À l'image de l'industrialisation, l'aménagement touristique est une construction européenne, ou plutôt une "co-construction » : bien des aménagements apparaissent en effet comme le résultat de négociations multiples et d'agencements situés qui croisent les désirs formulés par les touristes, les ambitions des promoteurs touristiques et l'intervention de toute une série d'intermédiaires (les médecins, les propriétaires, les hôteliers, les élus, les associations, les préfectures, les services 
déconcentrés de l'État, les cabinets d'expertise, les individus «frontière » aux appartenances et aux relations diverses, etc.). Les acteurs "locaux", avec toute la prudence qu'impose l'usage de ce terme, montrent un souci constant de plier les investissements et les aménagements à leurs ambitions de développement ou de valorisation patrimoniale. Cela s'opère pour la meilleure réussite au XIX ${ }^{\mathrm{e}}$ siècle, avec des résultats plus hasardeux en matière de sports d'hiver au XXe.

4 Un schéma fort original dans l'histoire économique se dessine par ailleurs: l'expérimentation très précoce d'une organisation communale et régionale de l'économie. Des communes, appuyées par des administrations diverses et parfois concurrentes (généralités d'Ancien Régime puis préfectures, Ponts et Chaussées et Mines), planifient leur urbanisme pour canaliser la spéculation immobilière, expérimentent des modes de gestion de biens productifs (des thermes jusqu'aux stations de ski en passant par les casinos). Le rôle de l'État central est surtout réglementaire, promotionnel, statistique, technique ; il n'est moteur d'aménagements que pendant une vingtaine d'années, entre les années 1950 et 1970. Ce sont sinon les communes, les départements, les intendances puis les préfectures et d'autres services déconcentrés qui impulsent et discutent de manière plus ou moins autonome les aménagements, du thermalisme aux sports d'hiver.

5 La logique même de l'aménagement touristique semble pouvoir être éclairée par le concept, emprunté à Michel Foucault, d'hétérotopie, cette utopie effectivement réalisée. Créer un espace qui permet d'échapper au quotidien semble en effet au principe de l'action sur l'espace. Cet angle d'attaque conduit à interroger non seulement le versant matériel de l'aménagement touristique, mais aussi son versant social, en étudiant les dispositifs qui ont concrètement été mis en place pour assurer le bon ordre et la sécurité dans les stations. Pour que l'espace touristique fonctionne comme une utopie effective, il a bien sûr fallu aménager l'espace, magnifier les eaux, la neige et les paysages; il a aussi fallu discipliner les populations locales en régulant les usages locaux, en contraignant les activités non touristiques en saison, en réglementant la somme de métiers informels. Ceux-ci ont longtemps permis d'intégrer de manière souple l'économie touristique aux autres activités, dans un système essentiellement pluriactif pour l'essentiel des populations locales. En effet, au-delà des grands propriétaires, hôteliers, médecins et loueurs de villas, toute une économie plus ou moins régulière anime les principales stations et permet à nombre de locaux de profiter des retombées des flux touristiques. Ce modèle semble se fragiliser en fin de XIXe siècle sous l'effet de la professionnalisation de l'offre, de la venue de travailleurs qualifiés saisonniers et de la mécanisation de certaines activités, comme celle des guides du fait du développement de l'automobile et des remontées mécaniques.

6 Les effets du tourisme sur l'environnement sont également abordés: très tôt, les urbanisations accroissent l'exposition aux risques naturels, artificialisent les sols, posent des problèmes d'assainissement, tandis que les excursions et les consommations diverses augmentent nettement la pression sur la biosphère. La chasse et la pêche en sont des exemples. Le tourisme n'est pas monolithique et, selon les acteurs, les lieux et les moments, il motive la protection de l'environnement comme son artificialisation, engendrant par là-même sa propre contestation. Cette question environnementale conduit aussi à interroger le partage de l'espace. Un changement de territorialité s'opère en effet avec l'apparition de nouvelles ressources et de nouveaux usages : de terroir de travail, les montagnes deviennent territoire de rêves et de loisirs. Leur 
manière de faire ressource change. Le tourisme transforme en fait la montagne - du moins certaines montagnes, comme celles du Luchonnais, des vallées du Gave ou d'Ossau - en patrimoine, au double sens du terme, à la fois héritage émotionnel commun à préserver et capital de base à exploiter. Cette question patrimoniale amène alors à étudier les liens entre le tourisme et la protection de la nature. Cette dernière n'a pas toujours été vécue comme un instrument de dépossession territoriale mais a pu être mobilisée localement pour défendre la ressource touristique (les paysages, les cascades, la pêche, les forêts, etc.) contre d'autres usages, parfois locaux, comme lorsque l'agropastoralisme a subi les foudres des associations et des municipalités touristiques en fin de XIXe siècle, parfois extérieurs, comme avec l'industrie électrique à partir des années 1900 et jusqu'à nos jours. Ces relations entre tourisme, industrie et agriculture sont fluctuantes et, plutôt qu'un modèle général, il faut retenir les modalités de négociation de partage de l'espace entre les activités.

7 Tandis que certaines communes touristiques les appelaient de leurs vœux afin de sauver de l'industrie leur patrimoine paysager, les parcs nationaux sont vus, à partir de la loi de 1960 qui les crée, comme une nouvelle menace de dépossession territoriale. Dans les années 1970, le cas du rejet du parc national des Pyrénées ariégeoises met en lumière ce que la protection de la nature contient de foncièrement politique - comme tout autre projet d'aménagement du territoire. Par les parcs nationaux, l'administration essaye de rationaliser l'usage du territoire national et d'assigner un rôle et une place aux collectifs humains et non humains qui y vivent : figurer (dans un but identitaire autant que commercial) les racines de la nation, racines que la nécessaire modernisation fait partout ailleurs disparaitre. Les parcs suscitent un ressentiment fort répandu quant à l'économie touristique : ils sont accusés de faire des façons d'être des spectacles pour les populations urbaines en mal de nature et de traditions. Le cas ariégeois montre aussi comment les services de l'État et, bientôt, les cabinets d'expertises ingèrent la rhétorique de la contestation pour mieux la dépolitiser, voire la "marchandiser ».

8 Un dernier apport se dégage de cette thèse. En remontant aux origines du phénomène et sans exclure les petites stations, il est permis de discerner des éléments qui ont pu discriminer les trajectoires des aires et stations touristiques. Cette histoire comparée, sans négliger, loin de là, la part fondamentale de la contingence, montre l'importance de la maîtrise foncière et de l'impulsion publique dans le lancement des stations. Aux $\mathrm{XVIII}^{\mathrm{e}}$ et $\mathrm{XIX}^{\mathrm{e}}$ siècles, cette action publique en faveur de l'exploitation touristique de l'environnement est permise par la mobilisation d'une autre ressource environnementale : les forêts communales, dont les coupes intensives pourraient bien être le facteur décisif du démarrage des grandes stations pyrénéennes, de Luchon à Cauterets. Pour les sports d'hiver, c'est l'évolution indéterminée et absolument imprévisible des pratiques qui déclasse les aménagements pionniers de Superbagnères et de Font-Romeu : ces dernières ont été construites à grands frais (privés cette fois) dans les années 1910 pour le ski de fond, le patinage et la luge, mais elles sont dépassées lorsque le ski alpin se répand dans les années 1930 puis, surtout, 1950, en parallèle de l'essor automobile - ce qui n'était guère prévisible au moment de leur construction. Ces exemples pionniers en France invitent en particulier à reconsidérer l'histoire traditionnellement faite des aménagements touristiques hivernaux, celle des " générations » successives de stations. L'étude de l'articulation entre les sports d'hiver et les activités touristiques plus anciennes, comme le thermalisme, montre que l'ancienneté, selon les cas, peut aussi bien être une force, par la renommée acquise et la 
capacité humaine et financière à anticiper le changement, qu'une faiblesse, par la pesanteur des intérêts constitués. Cette étude comparative et transfrontalière permet de restituer la part de contingence et les nombreuses divergences qui animent l'histoire, résolument non linéaire, des stations. Le récit historique doit veiller à restituer ce foisonnement d'expériences et à laisser ouvert le champ des possibles et de la création, dans le passé, le présent et a fortiori le futur.

9 Sans qu'il ne s'agisse aucunement d'une renonciation intellectuelle, cet examen des facteurs qui ont influé sur les trajectoires des stations pyrénéennes confirme l'indétermination essentielle de l'histoire. Aucun lieu, aucune société n'ont été et ne sont condamnés à quoi que ce soit. Leurs trajectoires se comprennent au croisement des controverses et des choix politiques, des dynamiques sociales, des héritages, des changements, des permanences et des contingences imaginaires et environnementales. Ces trajectoires, plutôt que d'offrir des modèles prédictifs pour le futur, permettent de voir comment, par le passé, on a répondu à des enjeux d'aménagement et de développement, qui continuent souvent à se poser, mais également de se rendre compte des multiples paris faits sur les futurs passés. Des paris qui sont autant d'expériences capables d'éclairer à présent les décisions, sans pour autant les abriter derrière le paravent quelconque de modèles immuables.

\section{AUTEUR}

\section{STEVE HAGIMONT}

ATER en histoire contemporaine, département d'histoire, FRAMESPA, CNRS, UMR 5136,

Université de Toulouse Jean Jaurès

Docteur et agrégé en histoire

steve.hagimont@univ-tlse2.fr 